\title{
Uso Racional de Antibióticos: uma experiência para educação em saúde com escolares
}

\author{
Rational Use of Antibiotics: \\ an experiment for the health education of schoolchildren
}

\author{
Bruna Werner Dandolini ${ }^{1}$ \\ Lilian de Bem Batista ${ }^{1}$ \\ Lúcia Helena Fernandes de Souza ${ }^{2}$ \\ Dayani Galato ${ }^{3}$ \\ Anna Paula Piovezan ${ }^{3}$
}

${ }^{1}$ Programa de Mestrado em Ciências da Saúde, Curso de Farmácia, Universidade do Sul de Santa Catarina (UNISUL). Av. José Acácio Moreira 787. 88704-900, Tubarão, SC.

bruwd@hotmail.com ${ }^{2}$ Unidade Acadêmica da

Saúde, Universidade do Sul de Santa Catarina

(UNISUL).

${ }^{3}$ Curso de Farmácia,

Programa de Mestrado em Ciências da Saúde,

Universidade do Sul de

Santa Catarina (UNISUL).
Abstract The promotion of the rational use of antibiotics can be conducted to ensure that health professionals and users become aware of its importance through educational activities. The scope of this paper was to report on the experiment of developing a tool for health education for primary school children on the rational use of antibiotics. The activities began by defining objectives, selecting the best strategy and planning activities with the guidance of teaching assistants in schools. After defining that the strategy to be adopted would be a comic strip, we worked together with the children on the steps of contextualization, sequence construction and its illustration. All the goals established for the story were achieved, since it represented aspects envisaged in the concept of rational use of medicines recommended by the World Health Organization. At the end, the story that best matched the intended purposes was selected to be turned into a comic book. The experience showed a way to develop health education activities in interaction with the community, using recreational workshops. Beside this, it made it possible to identify the limitations and potential for using education action strategies on other topics with children of the same age group.

Key words Health education, Child, Anti-bacterial agents
Resumo A promoção do uso racional de antibióticos pode ser realizada de forma a conscientizar profissionais de saúde e usuários através de ações educativas. Objetivo: Relatar a experiência de construção de um instrumento de educação em saúde sobre o uso racional de antibióticos com escolares do ensino fundamental. Métodos: As atividades iniciaram-se pela definição dos objetivos, seleção da melhor estratégia e programação das atividades com as assistentes pedagógicas das escolas. Após definir a estratégia a ser adotada como história em quadrinhos, trabalhou-se com as crianças nas etapas de contextualização, construção da sequência da história e sua ilustração. Resultados: Os objetivos previstos para a história foram alcançados, com a mesma retratando aspectos previstos no conceito de uso racional de medicamentos da Organização Mundial de Saúde. Obtiveram-se seis histórias e foi selecionada aquela que mais se adequou aos objetivos iniciais. Conclusão: A experiência demonstra uma maneira de desenvolver ações de educação em saúde em interação com a comunidade, utilizando oficinas lúdicas. Além disso, permitiu identificar limitações e potencialidades para a utilização de estratégias de educação em saúde, que podem ser realizadas com crianças da mesma faixa etária, sobre diferentes temas.

Palavras-chave Educação em saúde, Criança, Agentes antibacterianos 


\section{Introdução}

Para a Organização Mundial de Saúde o Uso Racional de Medicamentos é uma necessidade que vem sendo alertada já há algum tempo ${ }^{1}$, tendo em vista os problemas observados com o uso inadequado dos mesmos. De acordo com documentos oficiais desta organização ${ }^{2}$, em todo o mundo mais de $50 \%$ de todos os medicamentos são receitados, dispensados e vendidos de forma inadequada, enquanto a maioria da população mundial carece de acesso aos mesmos. Se de um lado este fenômeno contribui para o aumento dos gastos de recursos públicos com saúde, do outro lado traz prejuízos à população. Estes podem ser em decorrência do mascaramento de questões sociais do processo saúde/doença ${ }^{3}$, do aparecimento de reações indesejadas ${ }^{4}$ ou da ausência de resultados positivos para o tratamento. Neste último caso, um dos principais exemplos seria o do uso indiscriminado dos antibióticos, que tem como resultado uma maior resistência microbiana ${ }^{5}$.

No Brasil, o uso irracional dos medicamentos é fortemente influenciado por questões como a automedicação ${ }^{6}$ e a propaganda e publicidade tendenciosas ${ }^{7}$, que como consequência podem ocasionar intoxicações e até mesmo mortes ${ }^{8}$. Uma das estratégias capazes de impedir tal fenômeno, que é estimulada internacionalmente, é o de educação dos usuários sobre medicamentos ${ }^{9}$, sendo que esta medida pode ser aplicada tanto individual como coletivamente, e pode ser capaz de gerar mudanças de comportamentos e atitudes para a melhoria de condições de saúde. Estas ações de educação em saúde envolvem a aprendizagem de diferentes aspectos das doenças e integram o campo da promoção da saúde, levando em consideração os conhecimentos de determinada população, criando vínculos entre sua maneira de pensar e de agir no cotidiano, não apenas em situações de doenças ${ }^{10}$.

Resultados benéficos de campanhas desta natureza têm sido demonstrados em vários países, tanto na comunidade ${ }^{11}$ quanto nas escolas ${ }^{12,13}$. No Brasil, tais ações também foram empreendidas com resultados positivos entre as crianças, e dentre estas podemos citar aquelas realizadas com o objetivo de aumentar a competência no autocuidado da asma ${ }^{14}$ e de implementar hábitos para o manejo da obesidade ${ }^{15}$. Assim, dife- rentes órgãos de saúde incentivam sua prática nos mais diversos ambientes, como instrumento de transformação das práticas inadequadas de saúde.

Um destes ambientes pode ser a escola, pois a mesma é reconhecida como um espaço propício para tais atividades de promoção e prevenção, além de possuir uma responsabilidade social neste sentido ${ }^{16,17}$. Além disto, este tipo de ação encontra-se em consonância com o preconizado pelas diretrizes da meta 'saúde para o ano 2000' uma vez que visa à educação como forma de prevenção e de controle de problema de saúde, envolve diferentes setores da comunidade além da esfera da saúde, e promove a participação comunitária no planejamento dos mesmos ${ }^{18}$. Em concordância com o acima exposto, este estudo teve como objetivo relatar a experiência de desenvolvimento de um instrumento de educação em saúde com escolares do ensino fundamental sobre o uso racional de antibióticos.

\section{Materiais e métodos}

Para o desenvolvimento da estratégia educativa proposta, seguiu-se uma abordagem qualitativa baseada no relato de experiência ${ }^{19}$, na qual os pesquisadores e participantes representativos da situação ou do problema estão envolvidos de modo cooperativo ou participativo, orientada para a elaboração de diagnósticos, identificação de problemas e busca de soluções.

Foram sujeitos deste estudo as crianças regularmente matriculadas no quarto ano do ensino fundamental, de uma escola particular do município de Tubarão, ao sul de Santa Catarina, Brasil. Os procedimentos adotados para a execução deste estudo foram aprovados pelo Comitê de Ética em Pesquisa da UNISUL.

O grupo de trabalho envolvido no desenvolvimento destas atividades se constituiu de duas professoras e duas acadêmicas do Curso de Farmácia da Universidade do Sul de Santa Catarina (UNISUL), além da Assistente Pedagógica da Unidade Acadêmica (UnA) da Saúde desta instituição. Na escola, as atividades foram norteadas pela respectiva orientadora pedagógica.

As etapas adotadas para o desenvolvimento desta estratégia de educação em saúde estão apresentadas a seguir. 


\section{Descrição da Experiência}

\section{Etapas de Planejamento \\ - Sem a Participação das Crianças}

\section{Definição dos objetivos a serem}

\section{atingidos com a estratégia educativa}

Para a elaboração da história em quadrinhos, os autores selecionaram tópicos relativos ao Uso Racional de Antibióticos, com base no que é discutido na literatura acerca de os principais problemas encontrados em relação a esta classe de medicamentos. Utilizou-se como norteador o conceito da Organização Mundial de Saúde de que "O uso racional de medicamentos parte do princípio que o paciente recebe o medicamento apropriado para suas necessidades clínicas, nas doses individualmente requeridas para um adequado período de tempo e a um baixo custo para ele e sua comunidade" 9 .

Neste caso, estabeleceu-se que a história deveria abordar as seguintes questões: a prescrição do antibiótico por um médico, a aquisição do antibiótico apenas com a prescrição médica, o cuidado com a posologia prescrita para o antibiótico. Ao abordar estas questões, se pretendeu que a atividade desenvolvesse nas crianças as capacidades de: a) atribuir a profissional da área da saúde capacitado, neste caso o médico, a responsabilidade pela prescrição de antibióticos; b) identificar que a compra de antibióticos na farmácia deve ser feita apenas sob prescrição médica; e, finalmente, c) identificar que o tratamento eficaz com antibióticos depende da utilização dos mesmos na posologia adequada e determinada pelo médico prescritor.

\section{Seleção da estratégia educativa}

A escolha do tipo de estratégia educativa a ser desenvolvida no estudo levou em consideração a orientação pedagógica para a faixa etária envolvida, além das características da turma alvo de alunos $^{20}$. Desta forma, optou-se pela elaboração de uma história em quadrinhos, que possuiria os benefícios de envolver todas as crianças da turma, bem como de poder ser executada dentro de um período de tempo convidativo às crianças.

\section{Implementação}

\section{- Envolvendo a Participação das Crianças}

A elaboração da história em quadrinhos foi realizada em duas etapas, em dois encontros de cerca de uma hora e meia cada. As reuniões com as crianças só aconteceram após minuciosa or- ganização das atividades, que foram planejadas em conjunto com o orientador pedagógico da escola. Nesta organização estava contemplada a forma de abordagem das crianças a fim de atingir os objetivos previstos para cada uma das sessões.

\section{Definição da Sequência \\ da História em Quadrinhos}

$O$ primeiro encontro foi realizado num espaço da escola denominado de Sala de Vivências, e teve a finalidade de apresentar o trabalho às crianças e coletar dados da realidade das mesmas, transcrevendo-os para a sequência da história a ser contada em acordo com os objetivos estabelecidos para a mesma. Nesta etapa, estiveram presentes 23 alunos, do total de 26 matriculados na turma. O grupo de autores introduziu a problemática apresentando um teatro, com cerca de dez minutos de duração, como forma de contextualização do tema.

Terminado o teatro, abriu-se espaço para retirar dúvidas das crianças sobre o assunto e solicitou-se o auxílio das mesmas para a construção da sequência da história em quadrinhos. Um moderador conduziu o questionamento aos alunos com relação à sequência da história em quadrinhos, bem como das características físicas dos personagens e dos ambientes da história. Os dados foram sendo registrados por uma segunda pessoa, que também auxiliou o moderador no direcionamento das questões.

\section{Ilustração da História em Quadrinhos}

$\mathrm{O}$ segundo encontro foi idealizado com o objetivo de serem realizadas as ilustrações da história em quadrinhos, cuja sequência foi elaborada na semana anterior. Estiveram presentes 24 crianças, que foram divididas em seis equipes de quatro alunos. Para cada grupo foi distribuído material de desenho e pintura, bem como um resumo do encontro anterior que relembrava a sequência e os personagens, assim como as características físicas dos mesmos e dos ambientes da história. Ao final do encontro, obtiveram-se seis ilustrações diferentes para a história, contendo os desenhos e as falas.

\section{Análise dos Dados - Avaliação das Histórias}

Para avaliação dos resultados obtidos com a ilustração da história em quadrinhos pelas crianças, levou-se em consideração primeiramente os critérios definidos com os estudantes, relacionados à caracterização dos personagens e às fa- 
ses da história. Em segundo lugar, se os desenhos e as falas refletiam os objetivos traçados inicialmente para esta ferramenta de educação em saúde, com relação ao Uso Racional de Antibióticos, baseado no conceito da Organização Mundial de Saúde ${ }^{1}$.

\section{Considerações Éticas da Pesquisa}

Os procedimentos adotados para a execução deste estudo foram aprovados pelo Comitê de Ética em Pesquisa da UNISUL.

Os resultados desta experiência foram devolvidos às crianças participantes e socializadas com os demais alunos da escola.

\section{Resultados}

Como mencionado no relato da construção da ferramenta, o encontro inicial com as crianças da primeira escola começou com a apresentação de um teatro, com o objetivo de criar um contexto para a participação das mesmas nas atividades previstas. Após a apresentação do teatro pelo grupo de pesquisadores, algumas questões foram levantadas pelos alunos: "E se o farmacêutico vender o antibiótico sem receita?", "Por que tenho que tomar meu remédio uma hora antes de comer?", "E vírus, é tratado com o quê?". Os autores responderam às perguntas e iniciaram $o$ trabalho de construção da história em quadrinhos, conforme segue.

\section{Sequência da História \\ e Características dos Personagens}

A sequência e os personagens da história em quadrinhos ficaram assim estabelecidos: 1) em casa, uma criança está doente e pede um 'remédio' à mãe; 2) a mãe explica que só o médico poderia saber qual o melhor medicamento para ela e a leva ao consultório; 3 ) no consultório, o médico examina a criança, explica que ela está com uma infecção e prescreve um antibiótico; 4) na farmácia, o farmacêutico exige a prescrição médica para o antibiótico e explica a importância de usar corretamente o medicamento; 5) novamente em casa, a criança está brincando e não quer tomar o antibiótico, a mãe insiste, lembrando o que o farmacêutico informou e a criança o aceita; 6) com o tratamento, a criança melhora da doença e vai comemorar num parque de diversões.

Quanto às características dos personagens envolvidos e dos ambientes onde a história se passa, quando houve divergências entre os participantes foi solicitada a intervenção da professora que estava responsável pela turma para conduzir a discussão no sentido de que as crianças chegassem a um consenso. Obtiveram-se as seguintes respostas: o paciente seria uma menina, o cuidador seria a mãe, o paciente estaria apresentando sintomas como tosse, dor de garganta e gripe. Sobre as características dos personagens decidiu-se: o médico seria gordo, careca e usaria jaleco; em seu consultório haveria computador, instrumentos para exames e brinquedos, pois se tratava de um especialista para crianças. Quanto ao farmacêutico, seria um homem magro e calvo, que também usava jaleco.

As crianças também citaram muitos detalhes que poderiam estar representados nos quadrinhos, por exemplo, que o médico poderia ter um estetoscópio no pescoço e em seu consultório poderia haver um diploma na parede, um portaretratos sobre a mesa, entre outros.

\section{Produção das Histórias em Quadrinhos}

Conforme pode ser observado na Figura 1, as falas e as características dos personagens e ambientes da história que foram levantadas no encontro inicial se refletiram, de maneiras distintas, nas ilustrações que foram realizadas no segundo encontro pelos alunos. Nesta figura são apresentados desenhos de cada uma das seis histórias construídas, representando o consultório médico, mostrando semelhança entre os personagens envolvidos, características dos personagens e de suas falas.

\section{Representação dos Objetivos Previstos na História em Quadrinhos}

Com relação aos objetivos previstos inicialmente para a história em quadrinhos foram atingidos, uma vez que as ilustrações contemplaram, conforme pode ser visto nos desenhos apresentados na Figura 2: um médico examinando o paciente e prescrevendo o medicamento (painéis a e b), o farmacêutico exigindo a prescrição médica (painéis c e d), o paciente sendo lembrado sobre a importância do uso do medicamento na posologia e pelo tempo determinado pelo médico (painéis e e f).

Nas ilustrações desta figura, as falas que fortalecem estas ideias também estão em acordo com o conceito da Organização Mundial de Saúde ${ }^{1}$, conforme pode ser acompanhado a seguir. Nos painéis $a$ e $b$, com relação ao personagem médico, 
Ilustração do consultório médico nas diferentes histórias em quadrinhos construídas pelas crianças.

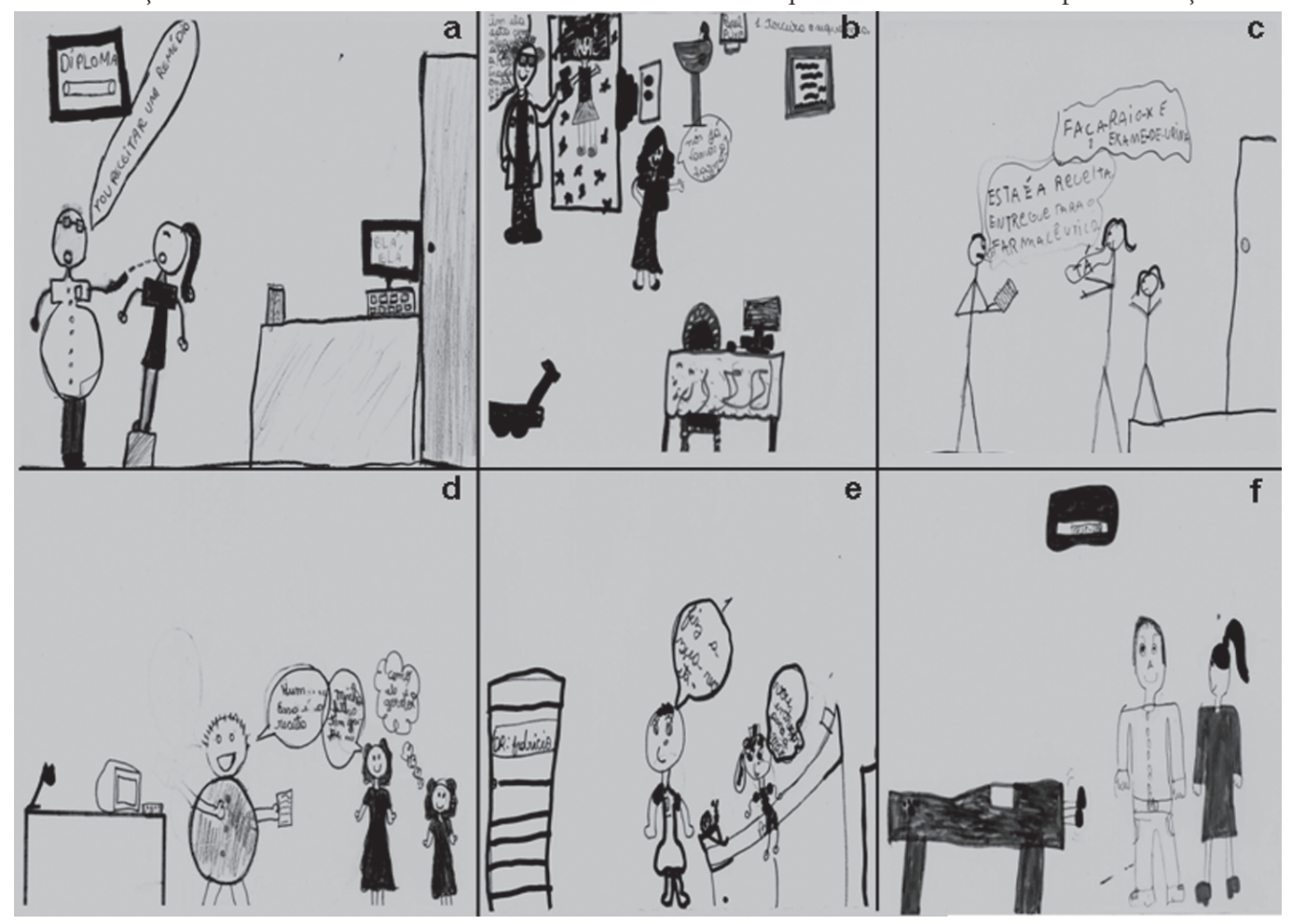

Figura 1. Representação das características dos personagens e ambientes da história em quadrinhos construída no primeiro encontro com os escolares.

as respectivas falas são: "Hum, ela está com infecção na garganta. Ela tem que tomar antibiótico" e "Hum... esta é a receita". Entende-se que estes quadros refletem, no primeiro caso a importância do diagnóstico pelo médico, e no segundo caso a competência deste profissional em prescrever o medicamento antibiótico. Já com relação ao personagem farmacêutico, nos painéis $c$ e $d$ os mesmos falam, respectivamente, "Me dá a receita” e "A receita, por favor”. Estas cenas levam à ponderação de que o medicamento antibiótico não pode ser vendido sem a prescrição médica. Finalmente, no painel $e$ a personagem mãe diz "Filha, hora do remédio" e a filha responde "Tá bem"; e, no painel $f$, o personagem médico diz "Menina, você tem que tomar remédio na hora certa e no tempo certo" e a filha responde "ok". Estas falas levam à sobre a importância do uso do medicamento na posologia adequada.

\section{Finalização das atividades}

Após a ilustração das histórias em quadrinhos pelas crianças, selecionou-se aquela que melhor representava os objetivos previstos para a mesma tanto em termos de sequência como da fala dos personagens. A mesma foi então digitalizada e diagramada no formato de uma história em quadrinhos, para que seja posteriormente avaliada a eficácia da mesma em campanhas de educação sobre uso racional de antibióticos com escolares da rede pública e privada do município envolvido.

Finalmente, como forma de devolução às crianças que participaram das atividades, promoveu-se um encontro final dos pesquisadores com as mesmas, durante uma das atividades de sala de aula. Nesta reunião, houve uma confraternização com distribuição de brindes na forma de materiais escolares (canetas, lápis e cadernos), bem como a devolução das histórias aos alunos, agora montadas em cartazes ampliados, que foram exibidos na escola para apreciação dos demais estudantes. Esta atividade foi previamente planejada com a orientadora pedagógica da escola e teve o objetivo de transformar num momento lúdico a ocasião de devolução dos resultados aos participantes. Destaca-se que a mesma 
Ilustração de partes das diferentes histórias em quadrinhos construídas pelas crianças.

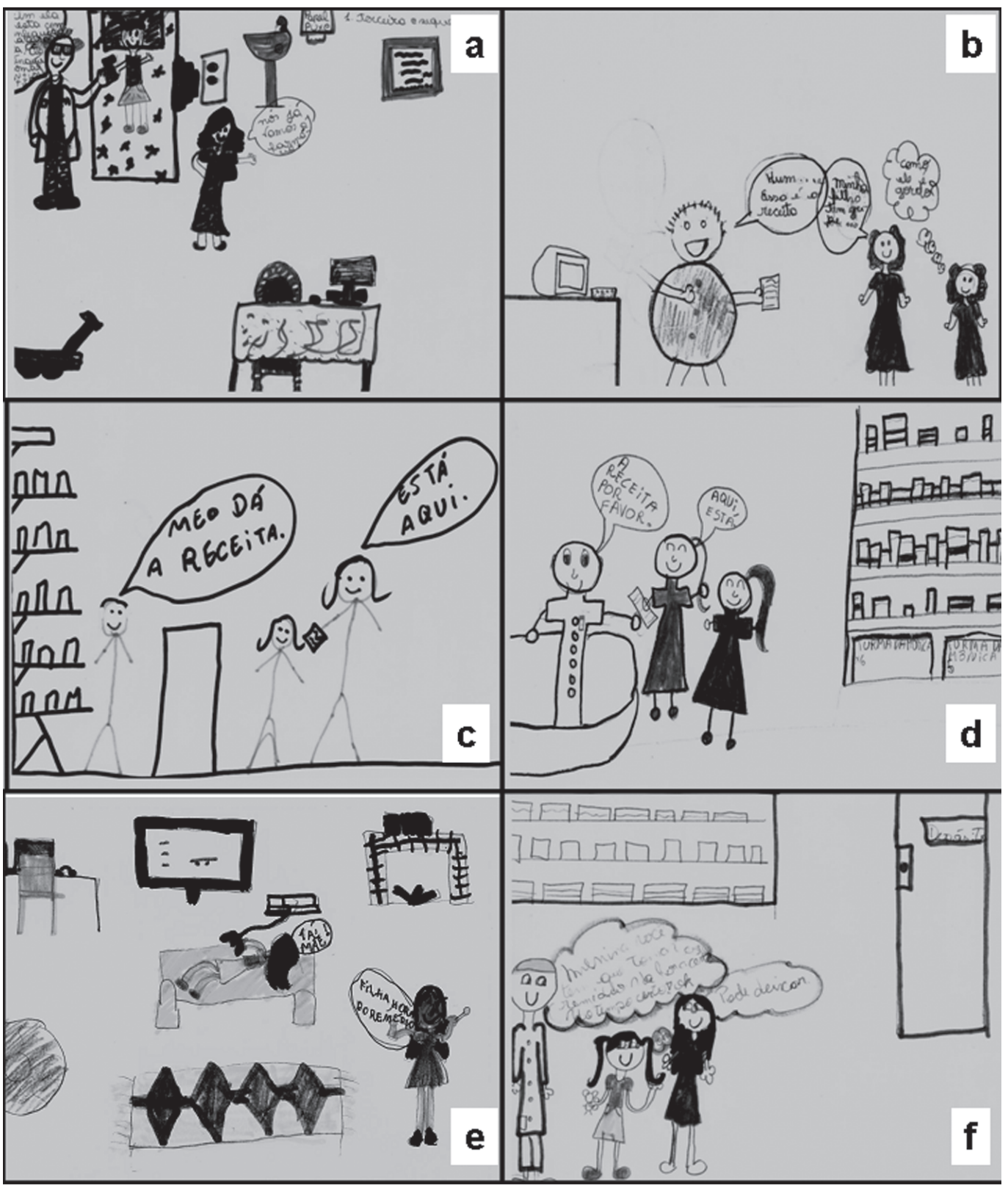

Figura 2. Representação de que todos os objetivos previstos para a estratégia educativa sobre uso racional de antibióticos foram alcançados.

foi planejada sem o conhecimento dos sujeitos da pesquisa (crianças), e que a obtenção dos resultados da pesquisa não esteve vinculada à participação das crianças neste evento.

\section{Discussão}

O presente trabalho demonstra a possibilidade de interação entre diferentes setores da comuni- dade na construção de uma estratégia educativa para crianças, numa área de grande importância para a saúde pública que é o uso racional de medicamentos. A educação em saúde com crianças vem sendo realizada com sucesso em diferentes países, sendo que no Brasil, na maioria das vezes, os trabalhos que avaliam esta prática o fazem a partir de atividades com grupos específicos, como aqueles com asma ${ }^{14}$ ou considerados obesos ${ }^{15}$. Sobre as ações em saúde na escola, podem ser 
relatados exemplos de resultados positivos de campanhas educativas para a prevenção de doenças infecciosas e parasitárias ${ }^{21,22}$; no entanto, até o presente momento não foram encontrados estudos abordando o tema dos medicamentos.

Sobre a importância de trabalhos realizados com crianças, no presente estudo, este público foi escolhido por duas razões, primeiro porque num futuro próximo esta será a geração responsável pela compra de medicamentos. Em segundo lugar, por acreditar-se que até mesmo na idade infantil estas podem servir de instrumento de transformação de hábitos na família e na sociedade, uma vez que também possuem capacidade crítica e reflexiva. Além disso, com relação à estratégia educativa realizada em nosso estudo na forma de uma história em quadrinhos, sabe-se que outras abordagens têm sido empregadas com sucesso por outros autores. Dentre estas podem ser citadas as peças de teatro ${ }^{23}$, manejo e observação do objeto de estudo ${ }^{24}$, palestras e carta$z^{22}{ }^{22}$, entre outros. Finalmente, a importância da realização de trabalhos desta natureza com crianças é fortalecida pelas campanhas promovidas recentemente por órgãos nacionais e internacionais com o objetivo de institucionalizar ações para reduzir a vulnerabilidade dos jovens às doenças sexualmente transmissíveis ${ }^{25}$. Ainda, está em acordo também com o preconizado pelo Ministério da Saúde, ao lançar a Estratégia das Escolas Promotoras de Saúde, dentro da Política de Promoção à Saúde ${ }^{26}$.

Com relação ao tema em questão, o uso indiscriminado de antibióticos é registrado tanto em nível hospitalar ${ }^{27}$ como na comunidade ${ }^{28}$. Este fato torna-se especialmente relevante, uma vez que no campo desta classe de medicamentos, as ações que são tomadas frente a um paciente podem repercutir em toda uma comunidade, principalmente no tocante à resistência bacteriana ${ }^{29}$, podendo acarretar em danos ainda mais graves aos indivíduos ${ }^{30}$.

Quanto às limitações do estudo, em primeiro lugar cabe lembrar que no presente trabalho não foi possível desenvolver uma reflexão mais extensa e crítica sobre a problemática do uso racional de medicamentos com as crianças envolvidas, anteriormente à construção da história em quadrinhos; o assunto foi abordado sob um conjunto de tópicos selecionados previamente pelos pesquisadores e contextualizado na forma de teatro.
A importância da inter-relação entre pesquisadores e comunidade, em ações que partem das reais necessidades destes últimos é muito bem comentada por Cyrino e Pereira ${ }^{31}$. Outra situação que merece consideração foi o fato de que as crianças reproduziram na história, parcialmente, a sequência do teatro apresentado pelo grupo de trabalho para introduzir as atividades. Em nossa opinião, contudo, isto reforça a ideia de outros autores, de que esta abordagem na forma de peça teatral também seja uma estratégia útil para a realização de campanhas educativas em saúde ${ }^{32}$. Finalmente, por tratar-se de um relato de experiência sobre a construção de uma estratégia educativa, os resultados atuais não nos permitem refletir mais amplamente sobre a possibilidade da mesma em promover mudanças na prática do uso racional de antibióticos, que segundo Tripp ${ }^{33}$ deve constituir-se em etapa finalizadora do processo de pesquisa-ação. No entanto, novos estudos estão sendo conduzidos pelo grupo com este objetivo, obedecendo metodologia mais adequada à avaliação de eficácia desta estratégia educativa, já que conforme Franco ${ }^{34}$, uma das características deste tipo de pesquisa é a continuidade das ações que permitam o aprofundamento e a apreensão de conhecimento por parte dos pesquisadores e dos participantes envolvidos.

Em conclusão, através desta experiência foi possível desenvolver uma estratégia de educação em saúde com escolares de ensino fundamental, na forma de história em quadrinhos. A utilidade da mesma para o conhecimento deste público foi constatada, uma vez que as histórias retrataram pontos importantes dentro do conceito de uso racional de medicamentos tais como seleção adequada do fármaco pelo médico, a venda do medicamento apenas sob prescrição e a utilização do mesmo dentro da posologia adequada.

A partir destas considerações, os pesquisadores percebem evidências para o estímulo às ações multidisciplinares desenvolvidas por profissionais de saúde e da área acadêmica junto às escolas, como forma de implantar a educação em saúde sobre diferentes temas. Além disso, sugerem que sempre que possível os pesquisadores possam projetar suas ações a partir de reflexões com os grupos a serem envolvidos, acerca de suas necessidades, com o tempo necessário à observação, ao planejamento, à implantação e à avaliação de resultados. 


\section{Colaboradores}

BW Dandolini, LB Batista, LHF Souza, D Galato e AP Piovezan participaram igualmente de todas as etapas de elaboração do artigo.

\section{Agradecimentos}

Agradecemos à orientadora pedagógica Mariléia Mendes Goulart e à farmacêutica Thaís dos Santos Corrêa.

\section{Referências}

1. Organización Mundial de la Salud (OMS). Conferencia de Expertos Sobre Uso Racional de los Medicamentos. 1985; Nairobi, Kenia. Ginebra: OMS; 1986.

2. Organización Mundial de la Salud (OMS). Promoción del uso racional de medicamentos: componentes centrales. Perspectivas políticas sobre medicamentos de la OMS sobre medicamentos. Ginebra: OMS; 2002.

3. Soares JCRS, Camargo KR. A autonomia do paciente no processo terapêutico como valor para a saúde. Interface Comunic Saúde Educ 2007; 11(2):65-78.

4. Pfaffenbach G, Carvalho OM, Bergsten-Mendes G. Reações adversas a medicamentos como determinantes da admissão hospitalar. Rev Assoc Med Bras 2002; 48(3):237-241.

5. Howard DH, Scott RD, Packard R, Jones D. The Global Impact of Drug Resistance. CID 2003; 36(Supl. 1):4-10.

6. Arrais PSD, Coelho HLL, Batista MCDS, Carvalho ML, Righi RE, Arnau JM. Perfil da automedicação no Brasil. Rev Saude Publica 1997; 31(1):71-77.

7. Luchessi AD, Marçal BF, Araújo GF, Uliana LZ, Rocha MRG, Pinto TJA. Monitoração de propaganda e publicidade de medicamentos: Âmbito de São Paulo. Rev Bras Cienc Farm 2005; 41:345-349.

8. Matos GC, Rozenfeld S, Bortoletto ME. Intoxicações medicamentosas em crianças menores de cinco anos. Rev. Bras. Saude Mater. Infant. 2002; 2(2): 167-176.

9. Organização Pan Americana de Saúde (OPAS). Relatório de Oficina de trabalho. Uso Racional de Medicamentos na Perspectiva Multiprofissional. Brasília: OPAS; 2007.

10. Schall VT, Struchiner M. Educação em saúde: novas perspectivas. Cad Saude Publica 1999; 15(Supl. 2):4-6.

11. Oliveira TBM, Presoto LH. Eficácia de um programa de promoção da saúde em infantes de préescola na cidade de Anápolis, Goiás. Cien Saude Colet 2009; 14(5):1891-1902.

12. Darnaud R, Dato MI. Educación para la salud en escuelas argentinas: concurso de plástica como actividad motivadora. Rev Panam Salud Publica 2009; 25(2):181-187.

13. Lizardo JMC, Rodríguez-Morán M, Guerrero-Romero F. El juego como alternativa para la enseñanza de conceptos básicos de salud. Rev Panam Salud Publica 2001; 9(5):311-314. 
14. Santana LFSCA, Bastos RCS, Martinez-Silveira MS, Souza LSF. Intervenções educativas em asma na infância: uma revisão analítica da literatura. J Bras Pneumol 2005; 31(5):445-458.

15. Mello E, Luft V, Mayer F. Atendimento ambulatorial individualizado versus programa de educação em grupo: qual oferece mais mudança de hábitos alimentares e de atividade física em crianças. $J$ Pediatr 2004; 80(6):468-474.

16. Silva CMC, Meneghim MC, Pereira AC, Mialhe FL. Educação em saúde: uma reflexão histórica de suas práticas. Cien Saude Colet 2010; 15(5):2539-2550.

17. Figueiredo TAM, Machado VLT, Abreu MMS. A saúde na escola: um breve resgate histórico. Cien Saude Colet 2010; 15(2):397-402.

18. Pan-American Healyh Organization (PAHO). Declaration of Alma-Ata. International Conference on Primary Health Care, Alma-Ata, USSR, 6-12 September 1978 [Internet site]. [accessed 2010 Jul 14]. Available from: http://www.paho.org/english/dd/ pin/alma-ata_declaration.htm

19. Minayo MCS. O desafio do conhecimento: pesquisa qualitativa em saúde. $8^{\text {a }}$. ed. São Paulo, Rio de Janeiro: Hucitec, Abrasco; 2004.

20. Anastasiou LGC, Alves LP. Processos de ensinagem na universidade: pressupostos para as estratégias de trabalho em aula. $6^{\text {a }}$ ed. Joinville: Universidade da Região de Joinville; 2006.

21. Schall VT, Pinto AG, Malaquias MLG, Santos MG. Educação em saúde em escolas pública de $1^{\circ}$ grau da periferia de Belo Horizonte, MG, Brasil: I - avaliação de um programa relativo à esquistossomose. Rev Inst Med Trop Sao Paulo 1993; 35(6):563-572.

22. Uchôa CMA, Serra CMB, Magalhães CM, Silva RMM, Figliuolo LP, Leal CA, Madeira MF. Educação em saúde: ensinando sobre a leishmaniose tegumentar americana. Cad Saude Publica 2004; 20(4): 935-941.

23. Pereira B, Silva KSS, Souza RP. Um cidadão não nasce grandão. Citizens in the Making: a contribution to children's environmental health education. Saude soc 2009; 18(Supl. 2):93-95.

24. Rancich AM, Ferrarini SO, Palma NH. Experiencia de enseñanza-aprendizaje: cambio de creencias y conductas en el manejo del agua de consumo. Rev Saude Publica 1989; 23(3):183-188.
25. Brasil. Ministério da Saúde (MS), Ministério da Educação, United Nations Educational, Scientific and Cultural Organization (UNESCO), United Nations Children's Fund (UNICEF), United Nations Population Fund (UNFPA). II Mostra Nacional Saúde e Prevenção nas Escolas. Brasília: MS; 2007.

26. Brasília. Ministério da Saúde (MS). A educação que produz saúde. Brasília: MS; 2005.

27. Souza HP, Vilhordo DW, Breigeiron R, Alessandretti MB, Dotti E, Silva TGS. Auditoria no uso de antimicrobianos em enfermaria cirúrgica. Rev Col Bras Cir 2008; 35(4):216-220.

28. Rauber C, Feltrin MR, Piovezan AP. Evaluation of antibiotics dispensing profile in Tubarão, Santa Catarina, Brazil. Braz J Pharm Sci 2009; 45(4):787-793.

29. Koch CR, Ribeiro JC, Schnor OH, Zimmermann BS, Müller FM, D’Agostin J, Machado V, Zhang L. Resistência antimicrobiana dos uropatógenos em pacientes ambulatoriais, 2000-2004. Rev Soc Bras Med Trop 2008; 41(3):277-281.

30. Spiandorello WP, Faintuch J, Ribeiro GT, Karkow FJ, Alvares JO. Use of multiple antimicrobial drugs by clinical patients: a prognostic index of hospital mortality? Clinics 2006; 61(1):15-20.

31. Cyrino EG, Pereira MLT. Reflexões sobre uma proposta de integração saúde-escola: o projeto saúde e educação de Botucatu, São Paulo. Cad Saude Publica 1999; 15(Supl. 2):39-44.

32. Souza WA, Vilas Boas OMGC. Orientação sobre o uso de vitamina A na saúde escolar: comparação de técnicas pedagógicas. Cien Saude Colet 2004; 9(1):183-190.

33. Tripp D. Pesquisa-ação: uma introdução metodológica. Educação e Pesquisa 2005; 31(3):443-466.

34. Franco MAS. Pedagogia da Pesquisa-Ação. Educação e Pesquisa 2005; 31(3):483-502.

Artigo apresentado em 22/03/2011

Aprovado em 05/04/2011

Versão final apresentada em 14/04/2011 